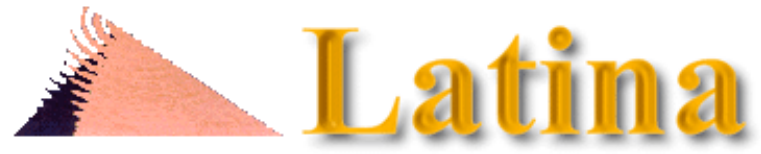

Revista Latina

de Comunicación Social - 62

enero - diciembre 2007

Edita: LAboratorio de Tecnologías de la Información y Nuevos Análisis de Comunicación Social Depósito Legal: TF-135-98 / ISSN: 1138-5820

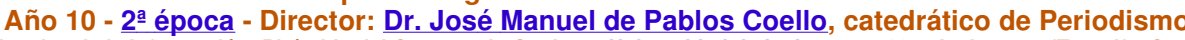

Facultad de Ciencias de la Información: Pirámide del Campus de Guajara - Universidad de La Laguna 38200 La Laguna (Tenerife, Canarias; España) Teléfonos: (34) $922317231 / 41$ - Fax: (34) 922317254

Investigación

FORMA DE CITAR ESTE TRABAJO EN BIBLIOGRAFÍAS, SEGÚN LA APA:

Pérez Martínez, V. M. (2007). Algunos aspectos deontológicos en la cobertura de informaciones científicas Estudio de caso: "Crisis volcánica" del Teide . Revista Latina de Comunicación Social, 62. Recuperado el x de xxxx de 200x, de http://www.ull.es/publicaciones/latina/200709Perez_Martinez.htm

[Revisora / revisor : Este artículo estudia un caso particular y extrae conclusiones muy pertinentes que sirven para la reflexión sobre las carencias profesionales de la actividad periodística en cuestiones que necesitan mucha mayor atención y responsabilidad social]

\title{
Algunos aspectos deontológicos en la cobertura de informaciones científicas Estudio de caso: "Crisis volcánica” del Teide
}

Artículo recibido el 10 de febrero de 2007 Sometido a pre-revisión (Comité de Redacción) el 14 de febrero de 2007 Enviado a revisión el 18 de febrero de 2007 Aceptado el 25 de abril de 2007 Publicado el 30 de abril de 2007

Mgs. Víctor Manuel Pérez Martínez @ [ C. V. ]

Doctorando de la Facultad de Ciencias de la Información

Universidad de La Laguna, ULL, ES

vmperezm@ull.es

Resumen: El artículo es el resultado de un trabajo de investigación enfocado en algunos aspectos deontológicos del periodismo científico. En primer lugar hace una revisión teórica sobre ciencia, periodismo, divulgación, internet y periodismo científico incluyendo algunas reflexiones sobre los principios deontológicos en el tratamiento de las fuentes informativas en las informaciones sobre ciencia y tecnología. Estos planteamientos sirven de base para la investigación en donde se plantea un análisis de la cobertura informativa sobre una presunta "crisis volcánica" en el Teide durante los años 2004-2005 realizada por el diario digital tinerfeño El Día. El trabajo requirió la revisión del material publicado en este diario y el posterior análisis de contenido a partir de las categorías establecidas. Se utilizó la versión digital con la finalidad de visualizar el alcance de las informaciones publicadas, además de ser cotejadas con su versión impresa, de cobertura local pero dirigido al público más afectado por las informaciones. Los resultados ofrecen algunos cuestionamientos sobre la forma cómo se realiza la cobertura informativa en un tema de interés local, nacional e internacional por las repercusiones que en el ámbito social, económico y turístico (principal sector industrial de Tenerife) puede tener este tipo de noticias.

Palabras clave: Periodismo científico - Internet - Deontología - Ciencia - Teide - Tenerife.

Abstract: The article is the result of an investigation which is focussed on some deontological aspects of the scientific journalism. In the first place it gives a theoretical vision about science, journalism, internet and including some reflections about the deontological principles in handling the information about science and technology. This focus is useful as it forms the base of an investigation where we deal with the information about a possible "volcanic crisis" in El Teide during the years 2004-2005 done by the digital newspaper" El Dïa" a canarian newspaper from Tenerife. The work required the revision of the information which was published and a followed analysis of its context. It was used the digital version with the purpose of visualizing the news which was published. It was also compared with a printed version, with local cover but divulged the information to the public who was most affected by this particular news. The results give rise to some questions regarding how the information is given to a topic which is of local interest as well as national and international interest due to the repercussions in the social, economical and tourist field (the tourist field is the main industrial sector in Tenerife) by receiving this type of news.

Key Words: Scientific journalism - Internet - Deontology - Science - Teide - Tenerife

Sumario: 1. Introducción. 2. Reflexiones sobre ciencia y periodismo científico. 3. Divulgación y periodismo. 4. Algunas reflexiones sobre el ámbito de la deontología. 5.Las fuentes en la deontología del periodismo. 6. Aspectos metodológicos. 7. Resultados de la investigación. 8. Características del "emisor" y de las "fuentes informativas" . 9. Las fuentes informativas principales. 10. Conclusiones. 11. Bibliografía. 12. Notas. 


\section{Introducción}

"Welcome to Terrorife" fue el titular de un artículo, del diario escocés Daily Record, refiriéndose al ambiente de tensión que se estaba generando en la isla de Tenerife por los movimientos sísmicos y las alteraciones en los valores normales de la composición de los gases volcánicos del Teide; estos datos podían indicar una posible erupción volcánica. El artículo, realizado por Michael Christie[1], hacía mención especial a la seguridad de los turistas que tendrían previsto visitar esta isla, aspecto que cobra importancia si tomamos en cuenta que desde Escocia viajan a Tenerife alrededor de 300.000 turistas a año. Unas de las afirmaciones más sensacionalista fue asegurar que existía un $80 \%$ de probabilidad de que ocurriera la erupción. Citaba las declaraciones de un portavoz de la Association of British Travel Agents, el cual señalaba que si había cualquier riesgo para sus clientes los turistas volverían inmediatamente o simplemente no viajarían a Tenerife. Estas ideas eran avaladas citando las declaraciones de fuentes expertas en el tema como Joan Martín, Ramón Ortiz y Nemesio Pérez. La erupción no ocurrió, pero, en el ámbito internacional, la alerta estuvo presente.

Esta alarma no solo se generó fuera de España. En el diario madrileño El País encontramos un artículo titulado, "Crisis volcánica en Tenerife" con el siguiente lead:

"Tenerife se enfrenta desde el pasado mayo a la primera crisis volcánica en casi un siglo, desencadenada por multitud de terremotos de escasa magnitud. Los expertos coinciden en que la probabilidad de una erupción es muy baja. Pero la unanimidad llega poco más allá. Las fuertes discrepancias entre algunos vulcanólogos respecto a la percepción del riesgo y gestión de la crisis han saltado a los medios de comunicación y han causado confusión y aturdimiento, admite uno de los miembros del comité científico asesor, que ha anunciado su dimisión"[2].

Estos dos artículos, al igual que otros publicados en diarios locales y nacionales[3], constituyen un ejemplo de cómo se están procesando las informaciones de carácter científico en algunos medios de comunicación social. ¿Cuál fue realmente la noticia sobre el Teide? ¿Fueron las fuentes cómplices, conscientes o inconscientemente de una política editorial por desacreditar la importancia turística de Tenerife? O quizás, ¿la falta de experiencia en el manejo de las fuentes periodísticas científicas por parte de los periodistas impidió ofrecer una información veraz? La respuesta a estas preguntas requiere de un estudio con detenimiento sobre el manejo de la información científica por parte de los profesionales de la comunicación social, la responsabilidad de los científicos al momento de ofrecer información y la política editorial de los medios de comunicación sobre temas de ciencia.

\section{Reflexiones sobre ciencia y periodismo científico}

La ciencia, entendida como el "conjunto de conocimientos obtenidos mediante la observación y el razonamiento, sistemáticamente estructurados y de los que se deducen principios y leyes generales"[4] ha sido el motor de algunos de los avances más importantes de la historia. Los planteamientos de los "hombres de ciencia" se habían analizado en espacios privados, en centros especializados, en reuniones, en congresos, en revistas y en libros; pero fueron los medios de comunicación social y más recientemente la utilización de las nuevas tecnologías, los responsables de la masificación de algunos mensajes científicos. En algunos casos con éxitos incuestionables, como el descubrimiento del Genoma Humano, que han convertido a la ciencia en un "nuevo dogma de fe" [5]; en otros, con resultados no muy satisfactorios para el mundo científico como la utilización de estos avances para la tecnificación de la guerra[6].

No toda la información científica es recibida igual por los individuos y grupos sociales y, en algunas ocasiones, simplemente desconocen su existencia aunque su presencia es cada vez mayor en los medios de comunicación social. Por otra parte, no toda la información de ciencia es "noticia" en la sociedad y no toda noticia científica es tal para los hombres de ciencia. ¿Un problema de lenguaje? Es cierto que los científicos son integrantes de la misma sociedad y, en algunas ocasiones, manejan un código tan particular dificultando un intercambio de información fluido y un acercamiento más allá de un descubrimiento espectacular o una nueva enfermedad identificada en un laboratorio. En esta realidad surge el periodista científico como el especialista que tiene la responsabilidad de recodificar el mensaje de las fuentes científicas y construir un mensaje para un público no especialista; entendiendo como periodismo[7], la captación y tratamiento, escrito, oral, visual o gráfico, de la información en cualquiera de sus formas y variedades.

El periodismo científico es un concepto reciente. Algunos, lo entienden como una disciplina o un conjunto de tecnologías. Es mejor definirlo como una "especialización que consiste en divulgar la ciencia y la tecnología a través de los medios de comunicación"[8]. No obstante, esta especialidad, genera otras interrogantes: ¿Qué es la ciencia? ¿Qué debemos entender como un hecho científico? ¿Una información no vinculada con la ciencia puede tratarse desde el ámbito del periodismo científico? Identificamos un campo amplio para el debate teórico, importante, para asumir desde otra perspectiva la actividad periodística de hoy.

Rithchie Calder señala: "la ciencia es la eterna interrogación de la naturaleza por el hombre"[9]. En este proceso por buscar la verdad, a través de métodos y técnicas, la humanidad ha evolucionado en el conocimiento de los hechos que le rodea, en las técnicas y en las tecnologías, aunque también en el mundo de la ciencia ha estado presente el debate ético y las implicaciones que han tenido sus teorías y descubrimientos para la humanidad, como ha sido el caso reciente de la clonación o el tratamiento de las células madres. El hombre está respondiendo cada día a los interrogantes que le plantea su entorno está en la búsqueda de respuestas, aunque aquellas que provienen de la ciencia no tienen "un carácter autoritario ni defiende dogma alguno"[10] pero son importantes para el desarrollo de la sociedad. En un mundo globalizado, cualquier avance científico puede convertirse en una noticia; la clave está: ies realmente la información que se publica sobre el hecho científico la verdadera noticia o la única noticia sobre el tema? Calder señala que los términos "ciencia" y "científico" han perdido su verdadero significado en la llamada era científica porque se ocultan detrás de la arrogancia, lo superficial y lo mágico; un fenómeno causado por los científicos, por los medios de comunicación y por la utilización de un lenguaje, en el cual emisor y receptor no coinciden[11]. Por otra parte, pareciera existir un abismo entre el conocimiento científico y su vinculación con la sociedad, concibiéndose la ciencia, aunque importante para el desarrollo, un mundo lejano a la realidad común del hombre. Es la visión metafórica del científico que se aísla de la realidad para buscar el conocimiento creando la sensación de que "la comunidad científica es inmune y ajena a las demandas de la sociedad"[12], un error que puede ser fortalecido por periodistas y científicos. Consideramos entonces que el periodismo científico tiene la misión de crear ese espacio común entre sociedad y científicos.

Manuel Calvo Hernando mantiene que los periodistas científicos tienen la misión de "explicar el Universo y acercar al pueblo 
el conocimiento"[13], otorgándole al ejercicio profesional una "misión casi sagrada". Entre las causas que han originado la brecha entre la sociedad y el mundo científico se encuentra la mitificación de la ciencia, el mundo de lo desconocido, los hechos sorprendentes e inexplicables para el individuo común. El periodismo científico es un espacio donde pueden unirse ciencia y sociedad, requiriéndose, del acercamiento de cada sector. El científico debe estar dispuesto a entender que su código debe ser comprensible por el receptor; la sociedad concebir la necesidad de educarse y asumir criterios para acercarse a la ciencia. En este contexto, los periodistas científicos deben asumir la importancia de su especificidad y razonar que se requieren de unos principios comunes a todas las especializaciones periodísticas, y a la vez, desarrollar estrategias propias que le permitan abordar y tratar la información científica.

\section{Divulgación y periodismo}

¿Qué es la divulgación científica? ¿Qué es el periodismo científico? Estos dos conceptos han generado diferentes posturas entre los investigadores y estudiosos del área. ¿Es el periodismo científico igual a la divulgación científica? Mariano Belenguer Jané plantea que hay dos concepciones fundamentadas en los conceptos de "información" y "divulgación". En el primer caso el periodista científico debe concentrarse en el hecho noticioso, sin que implique una actitud formativa sobre el tema científico; en el segundo caso el periodista científico no es solamente de carácter informativo sino que debe ser también formativo[14].

Divulgar significa "publicar, extender, poner al alcance del público algo"[15]. Para Roqueplo la divulgación es:

"toda actividad de explicación y difusión de los conocimientos, la cultura y el pensamiento científico y técnico, bajo dos condiciones, con dos reservas: la primera es que estas explicaciones y esa difusión del pensamiento científico y técnico sean hechas fuera de la enseñanza oficial o de enseñanzas equivalentes... La segunda reserva es que estas explicaciones extraescolares no tengan por fin formar especialistas, ni tampoco perfeccionarlos en su propia especialidad, ya que, por el contrario, reivindicamos completar la cultura de los especialistas fuera de su especialidad"[16].

Autores como Bonfil Olivera, consideran la divulgación científica como una "labor de difusión cultural"[17]. Manuel Calvo Hernando trasciende más allá de lo inmediato y señala:

"la divulgación de la ciencia se propone fines y objetivos de proyección social, perfeccionamiento de la democracia, proyección educativa y cultural, desarrollo integral y la búsqueda de soluciones inspiradas en la ética, como el uso del conocimiento científico como arma para impedir que en las sociedades se formen elites de expertos rodeadas de masas de analfabetos, aunque sepan leer y escribir" [18].

Desde esta perspectiva la divulgación tiene tres funciones: a) Función informativa: trata de hacer comprensible el ámbito de la ciencia; b) Función interpretativa: pretende buscar el sentido esencial de las teorías y principios científicos; c) Función de control: asumiendo en nombre de la sociedad una actitud crítica ante los grupos de poder. Desde esta percepción, el periodismo científico puede ser un tipo de divulgación científica pero no es su razón de ser. Es una herramienta para el fortalecimiento de la democracia, planteando un principio de "responsabilidad social" en la cobertura científica, en función de la igualdad entre los hombres: "El periodismo científico tiene la obligación social de hacer lo posible y lo imposible por que la ciencia y la tecnología no sirvan sólo para el enriquecimiento cultural y el beneficio práctico de algunas naciones o ciertas sociedades privilegiadas"[19].

En el ejercicio profesional del periodismo se aplican principios y técnicas para que la información, sobre un determinado hecho, llegue a la sociedad. En la divulgación científica el interés de que la sociedad conozca la ciencia es importante. En el periodismo, la esencia del mismo exige del periodista la constante búsqueda de la verdad sobre la información que se publica y sus repercusiones. Desde una perspectiva general, el periodismo es una forma de divulgar; pero la función del periodista trasciende al hecho de la divulgación. En esta línea, Dorothy Nelkin apunta: "Más que una simple fuente de información sobre la ciencia, la prensa juega un importante papel crítico"[20].

Martín Bonfil Olivera plantea diferentes tipos de divulgación[21], en donde menciona la "divulgación periodística o periodismo científico", definiéndola como aquella que le ofrece al público información e interpretación de los avances científicos enfocándose en las noticias. Desde otra visión, la divulgación científica busca enseñar, mientras que el periodismo científico busca la verdad en el campo de la ciencia, en donde no existen verdades absolutas. Javier Sampedro prefiere hablar de "verdades científicas establecidas", las cuales no implican en ningún momento que la misma evolución de los conocimientos científicos puedan modificar estas verdades. "No creo que sea correcto decir que Einstein invalida a Newton. Newton está dentro de las ecuaciones de Einstein. Einstein va más allá pero no invalida lo anterior"[22].

Por otra parte, el periodista científico no es un científico, es un periodista. La misión del científico es muy concreta y la de periodista también está definida. No podemos pretender que el periodista asuma el rol del científico porque no es su función y esta precisión es importante que sea considerada por los profesionales de la comunicación. Como señala Arístides Bastidas, el periodista científico "no es un sabiondo sino un profesional de la información que pone el ropaje común de todos los días al oscuro y ahuyentador lenguaje que usan los científicos"[23].

\section{Algunas reflexiones sobre el ámbito de la deontología}

"La información es como el agua. Nos es absolutamente necesaria para vivir. Pero, al mismo tiempo, podemos ahogarnos en ella por exceso o por mala canalización"[24]. Esta reflexión de Niceto Blázquez nos lleva a plantearnos dos interrogantes: ¿qué debemos informar? y ¿cómo debemos informar? Ambas preguntas son importantes en el ámbito del periodismo, pero en el caso del periodismo científico parecen ser indispensables. Nos encontramos con dos aspectos que hacen referencia directa al profesional del periodismo, a su forma de pensar y a su forma de actuar. La respuesta a cada una de ellas dependerá de variables como la actualidad, la proximidad, el interés, la oportunidad, el negocio, los intereses vinculados, entre otros. Como observamos, hay algunas variables de índole ético porque tienen que ver con los principios y las conductas del profesional; entramos en el campo de la deontología. Para Luka Brajnovic la deontología "abarca, por una parte, la profesión con todas sus consecuencias morales y proyecciones éticas sociales y, por la otra, los distintos aspectos de la conducta y conciencia humanas"[25].

Existen varios códigos deontológicos de la profesión periodística, incluso hay medios de comunicación que tienen su propio libro de estilo; esto supone una garantía para la sociedad si realmente los periodistas aplican los principios allí expuestos. Cada uno de estos instrumentos hace referencia a normas de actuación que pretenden garantizar un ejercicio profesional 
apegado a la defensa y a la búsqueda de la verdad. Con respecto a las fuentes informativas, hay planteamientos muy concretos, los cuales en general, suelen coincidir en varios instrumentos de este tipo. Pero ¿Cuál es nuestro interés por las fuentes informativas?

Además de limitar nuestro estudio por razones logísticas y técnicas, es en la "fuente" donde nacen los mensajes, siendo el periodista científico el responsable de codificar esa información. García del Castillo utiliza el concepto de "emisor-fuente": "Nos interesa conocer quién es quién. Es posible que nos interese, en un primer momento, quién es el emisor directo y, posteriormente, quien es el emisor, quién es la fuente, el inicio del proceso de la comunicación"[26]. En el campo del periodismo científico nos interesa el emisor (el periodista) y el tratamiento de la información; pero también nos interesan las fuentes utilizadas y cómo el emisor utiliza la información suministrada por la fuente, cuál es el valor que le otorga, su jerarquía, los cuestionamientos o respaldos de una fuente, entre otros aspectos. Las noticias científicas requieren de la utilización de fuentes especializadas, porque su mensaje es especializado.

\section{Las fuentes en la deontología del periodismo}

En general, la actividad periodística se inicia con la búsqueda de la información a través de una investigación enfocada en la búsqueda de datos veraces sobre los hechos o eventos que ocurren; siendo el apego a la verdad, un compromiso del periodista con la sociedad para la cual desempeña sus funciones. La investigación, comprende por parte del periodista la consulta de fuentes que le ofrezcan los datos requeridos para construir la información. En algunos casos, el propio periodista es testigo directo de los acontecimientos; pero en otras, como en la mayoría de las ocasiones ocurre con las informaciones científicas, hay eventos a los cuales no se puede acceder. El periodista no puede ser testigo de los movimientos de las placas tectónicas, ni tampoco de la explosión de una estrella en el universo. Además, hay hechos que aunque visibles, su explicación no está a la vista; en otros casos está presente la explicación pero es indescifrable por no conocer una determinada especialidad. Buscar la verdad para el periodista es un compromiso y su esencia. En todos los documentos que tratan sobre la deontología de la comunicación o de la información hay prácticamente consenso sobre el tratamiento ético de las fuentes informativas; principios que deben reflejarse en los textos informativos.

\section{Aspectos metodológicos}

La investigación estudió los datos procedentes de los mensajes periodísticos publicados en uno de los diarios digitales de la región, siendo el objeto de estudio el contenido de estos mensajes. Seleccionamos la técnica del análisis de contenido, entendiéndola, como "una técnica de investigación destinada a formular, a partir de ciertos datos, inferencias reproducibles y válidas que puedan aplicarse a su contexto"[27].

\subsection{Justificación del caso: Posible erupción volcánica del Pico Teide}

Son diversos los temas en el campo científico aunque algunos acontecimientos repercuten con mayor fuerza en los medios informativos y en la sociedad, especialmente si ellos afectan nuestro entorno. La probabilidad de una erupción volcánica en las Islas Canarias ha estado, y está, vigente en la región[28]; pero la inmediatez de una erupción del Pico Teide -el punto más alto de España con 3.718 metros-[29], comenzó a tener presencia en la prensa local y regional, a raíz del estudio realizado por la División de Medio Ambiente del Instituto Tecnológico y de Energías Renovables (ITER) el cual indicaba la existencia de registros de datos anormales con respecto a la emisión de los gases de este cono volcánico[30] y un aumento en el número de sismos en la región, presuntamente, relacionados con la actividad volcánica. Algunos investigadores plantearon como hipótesis que los cambios registrados podían estar vinculados a una erupción del Teide.

En el mes de mayo de 2004, a través del proyecto Guayota, se informó a la sociedad que el "semáforo volcánico" pasaba del "color verde" al "color amarillo"; es decir, la Comunidad Autónoma de Canarias se encontraba en "alerta permanente"[31] ante la posibilidad de una erupción. Algunos vulcanólogos señalaron que los indicadores no representaban ningún peligro porque los datos registrados no eran concluyentes para generar una situación de alarma, y en el caso que ocurriera la erupción, esta no tendría consecuencias graves para la isla de Tenerife; indicaron que la situación estaba generando una alarma innecesaria entre la población. Durante los diez meses que duró el estado de "alerta permanente" se identificaron eventos que fueron interpretados como la cercanía de una erupción; pero, ninguno de ellos pronosticaban para algunos científicos, una erupción inminente o una erupción de grandes proporciones. Afirmaron que eran eventos normales debido a las características propias de la región. No obstante, la información presentada en algunos medios de comunicación fue de alarma; incluso, se indicaba la existencia de una "crisis volcánica" por algunos medios, entre ellos, los diarios Canarias 7[32], El País[33] o el $A B C[34]$. La Nathional Geographic realizó un reportaje en el mes de mayo de ese año con el nombre de "Canarias, cita con el fuego". La información traspasó las fronteras españolas con un matiz aún más sensacionalista y así lo reflejó el Daily Record[35] con el título "Welcome to Terrorife". La preocupación se trasladó a la cotidianidad de los habitantes de las islas, especialmente en los municipios más cercanos al Teide, y junto a ella, la indignación de algunos sectores por el estado de alarma generado. Ambos sentimientos quedaron expresados en algunas letras de las tradicionales murgas del carnaval del año 2005[36]. En el Informe del 16 de Febrero de 2005 del Plan de Actuación Coordinada de Protección Civil ante una posible erupción volcánica en la isla de Tenerife[37], el "semáforo volcánico" volvió al "color verde"[38].

\subsection{Objetivos de la investigación}

El objetivo de nuestro trabajo fue: analizar, desde la perspectiva de la deontología del periodismo, el tratamiento de las fuentes informativas por parte del emisor en la cobertura realizada sobre una posible erupción de El Teide, a partir de los textos periodísticos informativos publicados en la edición digital del diario tinerfeño El Día (www.eldia.es) desde el 01 de enero de 2004 hasta el 31 de febrero de 2005.

Nos planteamos las siguientes preguntas de investigación: ¿Quién fue el emisor (autor) de los textos periodísticos? ¿Las informaciones publicadas contaron con fuentes informativas adecuadas? ¿Se contrastó la información ofrecida por las fuentes? ¿Se utilizaron tipos de fuentes propias del periodismo científico? ¿Cuáles son las relaciones entre las fuentes informativas y otras características de los textos periodísticos publicados? ¿Se aplicaron los principios deontológicos a las fuentes informativas utilizadas por el emisor?

La investigación incluyó las siguientes hipótesis:

a) Las fuentes informativas utilizadas reunieron las características para ser consideradas confiables, válidas y adecuadas 
b) Las informaciones sobre el tema fueron contrastadas.

c) Hay relaciones entre la utilización de las fuentes informativas y otras características de las informaciones publicadas.

d) Se aplicaron los principios deontológicos a las fuentes informativas en las informaciones publicadas.

e) Se utilizaron fuentes informativas propias del periodismo científico.

\subsection{Selección de la muestra}

El período de estudio comprendió desde el 01 de enero de 2004, mes cuando se informó del estudio del ITER; hasta, el 31 de febrero de 2005, mes cuando el semáforo volcánico pasó de "amarillo" a "verde". Escogimos este período por reunir los siguientes criterios: a) un tiempo previo a la presentación del estudio del ITER; b) la activación de la alerta volcánica con el cambio del semáforo del color verde al color amarillo (Mayo 2004); c) el aviso de la normalidad volcánica con el cambio del semáforo a color verde (Febrero 2005)

El estudio estaba centrado en el contenido de las informaciones publicadas sobre el tema seleccionado. No era una comparación de la cobertura realizada por los medios de comunicación social, por eso, se seleccionó un solo medio de comunicación social. Limitamos el estudio al diario El Día por reunir las siguientes características:

a) Es un medio que se publica en la isla de Tenerife, lugar, donde se encuentra el Teide convirtiendo la información de una posible erupción en "noticia" de mayor interés por las implicaciones humanas propias del tema, por cumplir con el principio de "proximidad" y el principio de "actualidad"[39].

b) El Día era el diario de mayor "tiraje" y de mayor "difusión" según la Oficina de Justificación de la Difusión[40]. Se excluyeron los suplementos especiales, revistas y encartes que se distribuyeron en el diario, aunque, durante el período de estudio no hubo ninguna publicación especial sobre el tema volcánico.

c) El Día tenía una versión digital, en la cual, publicaba diariamente los artículos aparecidos en su edición impresa.

Las unidades de análisis identificadas fueron los textos informativos publicados sobre una posible erupción volcánica del Teide[41] y que correspondan a los géneros informativos: "la noticia y la entrevista"[42].

Se establecieron las dimensiones, categorías (cuantitativas y cualitativas) y variables, diseñándose una base de datos documental en donde cada unidad de análisis tenía su propio registro. Una vez codificado los resultados, se utilizó el paquete informático SPSS para la obtención de las frecuencias, las asociaciones, las correlaciones y otras técnicas estadísticas señaladas por Krippendorff y que son utilizadas para el análisis de contenido de los medios de comunicación.

\subsection{Limitaciones}

La utilización de un solo medio para el estudio del tema no nos permitió establecer comparaciones y analizar las características de toda la información recibida por la población. No solamente nos referimos a los medios de comunicación social de la isla de Tenerife, sino los del Archipiélago e incluso de España, por ser un tema de importancia, proximidad y actualidad para la región.

Con respecto a las categorías y a las variables vinculadas con el ámbito de la deontología hay que considerar otros aspectos que no fueron estudiados como el conocimiento de los periodistas sobre el tema, valoración en la sala de redacción de las informaciones, acceso a las fuentes por parte de los periodistas, material existente, entre otros.

El estudio se centró en un solo tema de carácter científico, incluso, en un solo caso vinculado al vulcanismo; habría que ampliar el estudio a otros temas o a otros casos de la ciencia que fueron publicados en el mismo diario.

En el período de tiempo se hicieron presentes otras informaciones vinculadas a eventos naturales como el tsunami ocurrido el 26 de diciembre de 2004 en el océano índico[43], o incluso al mismo vulcanismo, aunque, no hacían mención al tema que estábamos estudiando. Es posible considerar que estas informaciones también contribuyeron a fortalecer el estado de alerta en la población con respecto a una eventual erupción del Teide.

\subsection{Ficha Técnica}

Periódico: Versión digital del diario El Día (www.eldia.es)

Ejemplares revisados: 422 ejemplares

Fechas: Desde el 01 de Enero de 2004 hasta el 31 de Febrero de 2005

Textos Periodísticos Informativos: 53

\section{Resultados de la investigación}

El estudio comprendió exclusivamente los textos periodísticos[44] correspondiente al género informativo (textos periodísticos informativos = TPI): noticia y entrevista. Se excluyeron los géneros de opinión y los "géneros anexos" según la clasificación realizada por Yanes Meza. No descartamos la necesidad de hacer un estudio global de todos los géneros para poder identificar otras conclusiones, pero nuestro interés se centró en la actividad informativa exclusivamente. Una vez realizada la selección, se registró un total de 53 TPI que mantenían relación con una posible erupción del Teide; 50 registros fueron del género "noticias" $(94,3 \%)$ y 3 registros fueron del género "entrevistas" $(5,7 \%)$.

7.1. Distribución por mes 
La distribución de los TPI por la categoría "mes de publicación" indicó el comportamiento esperado en la evolución normal de una noticia. El hecho noticioso surgió en mayo de 2004 y culminó en febrero de 2005. La erupción nunca ocurrió, los hechos corresponden a varios eventos que fueron considerados noticias y los cuáles tenían un hilo conductor: la posible erupción de Teide. Primero fue la publicación del estudio del ITER, después el cambio de color en el "semáforo volcánico", seguidamente las series de sismos que se registraron y por último, después de varios meses, otro cambio de color del "semáforo volcánico".

En los meses de enero de 2004 y febrero de 2004 se publicaron dos TPI sobre estudios que se estaban realizando en El Teide. El primero de ellos, con el título "El ITER estima que el Teide emite 77 kilogramos de compuestos volátiles al año"[45]; esta información no hacía ninguna mención directa a una erupción volcánica; la información se limitó a señalar la importancia de los gases volcánicos para la atmósfera. El segundo, con el título, "El proyecto Guayota informa de la actividad sísmica cada mes"[46], informaba sobre la emisión de un "informativo gráfico sobre la actividad sísmica y volcánica que se registra en Canarias", el cual estaría avalado por la División de Medio Ambiente del Instituto Tecnológico y de Energías Renovables (ITER), -organismo dependiente del Cabildo de Tenerife-, en colaboración con el Centro Geofísico de Canarias del Instituto Geográfico Nacional (IGN). La noticia no hace ninguna mención a una posible erupción volcánica, ni a cambios en la composición de los gases que emanan en el Teide.

Fue en mayo de 2004, cuando el número de TPI aumentó (22,6\%). La noticia en el aumento del número de sismos que se registraron en la isla de Tenerife motivó al Comité Científico de Riesgos Volcánicos (CCRV) a realizar un mapa de riesgos volcánicos. El titular de la primera noticia publicada en el mes de mayo de 2004 fue: "Canarias empezará a redactar el mapa de riesgos volcánicos a partir de junio"[47]. El siguiente texto informativo plantea por primera vez la posibilidad de una erupción: "Los vulcanólogos estiman la posibilidad de una erupción tranquila en Tenerife"[48]. Posteriormente se reunía e Comité Científico de Evaluación y Seguimiento de Fenómenos Volcánicos y se retomaba la normativa existente para Canarias sobre la planificación de un sistema de protección ante el riesgo volcánico[49]. Desde ese momento el tema de la erupción del Teide tomó preferencia en El Día fortaleciéndose la información por el cambio de color en el "semáforo volcánico"[50]:

"El director de Medio Ambiente del ITER, Nemesio Pérez, aseguró ayer al concluir su reunión con responsables públicos del noroeste y sus de la Isla que nos encontramos con el semáforo en amarillo. Puede que vuelva a verde o se ponga en rojo dijo-, pero, de momento, estamos en amarillo"[51]

La frecuencia de los TPI retomó presencia en el mes de septiembre de 2004, cuando se registró un aumento del número de sismos y alteraciones en los valores de los gases que emanan del Teide. Con respecto al contenido de este mensaje no hubo consenso entre los científicos de que se trataba de indicadores válidos para afirmar la existencia de un proceso de erupción; las informaciones seguían emitiéndose, teniendo como fuente en su mayoría el boletín semanal emitido por el ITER.

A partir del mes de septiembre de 2004 la frecuencia de los TPI comenzó a disminuir por la falta de informaciones distintas sobre el tema. En el mes de febrero de 2005 solamente se registró un TPI, en el cual indicaba la vuelta a la normalidad, con el cambio de color del amarillo al verde, del semáforo volcánico.

\subsection{Distribución por tema principal y secundario}

Establecimos como categorías unos temas con la finalidad de agrupar los mensajes que aparecían en los TPI. El primer "tema principal" con mayor frecuencia fue el de "Fenómenos relacionados con la actividad volcánica" $(49,1 \%)$ que agrupó aquellos TPI en los cuales el tema central hacía referencia a sismos y a cambios en la composición de los gases volcánicos. El segundo tema, con una mayor frecuencia como "tema principal" fue el de "Planes de prevención, emergencia, evacuación, entre otros" $(13,2 \%)$, el cual agrupó aquellas informaciones que tenían como preferencia la realización de estrategias para prevenir o actuar ante una eventual erupción del Teide. El tercer tema, con una mayor frecuencia como "tema principal" fue la "Posible erupción volcánica del Teide" (9,4\%), que agrupó aquellas informaciones que señalaban principalmente estudios, informaciones 0 análisis sobre una erupción del Teide. Hay otros temas que tuvieron jerarquía en los TPI, pero con una menor presencia.

En una reagrupación de categorías clasificamos los temas como: a) "Alerta", temas que hacen referencia a situaciones de alarma; b) "Normalidad", temas que hacen referencia a mensaje de tranquilidad, normalidad, prevención, entre otros. Los datos obtenidos fueron: Un $73,6 \%$ de los TPI asumieron temas relacionados con un ambiente de "alerta", mientras que el $26,4 \%$ trataron temas relacionados con un ambiente de "normalidad".

Los resultados no ofrecen un cambio significativo cuando analizamos la frecuencia de los "temas secundarios" en donde aparece en primer lugar el tema de una "Posible erupción volcánica del Teide" (29,6\%), seguida del tema "Fenómenos relacionados con la actividad volcánica" $(24,5 \%)$, y del tema "Planes de prevención, emergencia, evacuación, entre otros" $(10,2 \%)$

\subsection{Características de los titulares de los TPI}

Utilizamos las siguientes variables para estudiar las características de los titulares de los TPI tomando en consideración el "grado de información". La variable "informativo" corresponde a titulares que condensan lo esencial de la noticia "y sólo con su lectura el receptor queda informado con bastante precisión"[52]; la variable "apelativo" corresponde a titulares que mencionan el asunto, "pero no dicen exactamente lo que ha sucedido"; la variable "impacto", es cuando el titular "no ofrece ningún dato de la información, y contiene, en pocas palabras, un mensaje llamativo". Analizamos los datos y obtenemos que un $56,6 \%$ tenemos titulares en los TPI considerados "apelativos" y que solamente un $32,1 \%$ de esos titulares son informativos. Debemos también indicar que la variable "de impacto", que más se relaciona con el sensacionalismo, obtuvo el $11,3 \%$ de los titulares de los TPI. Es de suponer que la utilización de los titulares "apelativos" tienen la finalidad de motivar al lector leer la noticia completa.

\subsection{Características del lead de los TP}

Con respecto al modo de presentar los datos[53] en los lead de los TPI, encontramos que el $50,9 \%$ tienen la característica de "Directo", es decir, ofrece una información clara de la noticia. El 47,2\% tiene la característica de "Diferido", es decir, no hace referencia al contenido principal de la noticia, obligando al lector a seguir leyendo la noticia para conocer la información. Por 
último, solamente se identifico un registro con un lead de carácter "interpretativo", es decir: el periodista realizó un interpretación de los hechos ocurridos.

También analizamos los lead tomando como criterio los recursos narrativos empleados. En este caso obtuvimos que, en un $50,9 \%$ tenemos un lead tipo "descriptivo", es decir, explica los hechos en forma clara y precisa; en un $39,6 \%$ tenemos un lead tipo "cita", es decir, reproduce las palabras o comentarios de una de las fuentes señaladas en la noticia; y en un $9,4 \%$ tenemos un lead tipo "ambiente" porque describe el entorno donde surge la noticia.

\section{Características del "emisor" y de las "fuentes informativas".}

Recordemos que en el proceso de la comunicación las fuentes son aquellas utilizadas por el emisor (el codificador) para construir su mensaje. El emisor se convierte en fuente para el lector. Desde la teoría vinculada a la deontología sobre las fuentes informativas, el emisor (el periodista) es quien escoge, jerarquiza, estudia, analiza e interpreta los datos ofrecidos por la fuente. Es esta la razón por la cual, nuestro análisis contempla al "autor o firma" del texto periodístico (emisor) y las "fuentes" utilizadas.

\section{1. ¿ Quién es el emisor de los TPI?}

Esta pregunta tiene la siguiente respuesta con base a los datos: El 67,9\% es El Día; el 17\% es la agencia de noticias EFE; $5,7 \%$ son iniciales. Únicamente el 9,5\% de los TPI tenían firma completa. Estos resultados solamente nos permiten concluir que el responsable de la mayoría de las informaciones fue el diario El Día, sin poder otorgarle a ninguna persona en concreto la autoría de los textos. Caso similar ocurre con la agencia de noticias EFE, aunque pareciera existir la percepción de que la información procedente de agencias de noticias no requieren una autoría específica al tratarse de textos que sirven de base para la realización de los trabajos; pero en esta situación la autoría corresponde directamente a la $E F E$.

Al cruzar los resultados de la categoría "Autor-Firma" con los resultados de las otras variables, hay algunos datos que consideramos importante indicar y comentar.

\section{2. ¿Cuál es el lugar desde donde firman los autores de los TPI?}

En el caso de El Día (considerándolo como autor del texto periodístico), la totalidad de sus textos fueron desde Tenerife, al igual que aquellos emisores que firmaron sus notas y aquellos que solamente indicaron las iniciales. La diferencia la marcó $E F E$, al incluir como lugar a Fuerteventura (1 caso), Madrid (2 casos) y Las Palmas de Gran Canaria (3 casos); éste último dato coincide con el número de textos firmados en Tenerife ( 3 casos). Pudiéramos señalar que EFE utilizó fuentes que provenían de varios lugares geográficos para tratar la noticia que nos ocupa.

\section{3. ¿ Cuáles fueron los temas principales desde las fuentes?}

Los resultados tienen importancia, no tanto por su cantidad, sino por la relación de su frecuencia con respecto a la totalidad de TPI que cada emisor publicó. Es decir:

a) El Día, asumió como "tema principal" el de los "Fenómenos relacionados con la actividad volcánica" en el 58,3\% del total de los textos periodísticos publicados; como segundo "tema principal" identificamos el de "Planes de prevención, emergencia, evacuación" que representó apenas el 16,7\%.

b) EFE, tiene un menor número de TPI con solo nueve casos representando el $17 \%$ del total publicado. Como autor le otorgó preferencia al tema "Fenómenos relacionados con la actividad volcánica" en un 33,3\% de los TPI; y como segundo tema de preferencia "Posible erupción del Teide" que significo el 22,2\% de los textos con su autoría.

c) Con respecto a las "firmas completas", de los cinco casos, dos de ellos tomaron como tema principal la "Posible erupción volcánica del Teide", y uno de ellos asumió "No existe crisis volcánica".

\subsection{Fuentes utilizadas}

El número de fuentes utilizadas por el emisor nos permite señalar la posibilidad de equilibrio en la información publicada. El estudio indica una ausencia significativa del número de fuentes. En el $67,9 \%$ de los textos publicados se utilizó una sola fuente, dejando a un lado la posibilidad de contrastar la información ofrecida por la misma. Solamente el $30,2 \%$ de los casos utilizaron más de una fuente.

Otra relación corresponde al mes de publicación, en el cual encontramos que en los primeros meses de la noticia se publicaron informaciones con más de una fuente, en contraposición a las publicaciones con una fuente que obtuvieron una mayor frecuencia después del mes de mayo. En particular con este mes, cuando se originó la noticia, encontramos que de las once noticias, seis contaban con más de una fuente $(54,5 \%)$. Fue probable que la noticia motivara la consulta de varias fuentes en ese momento ante la novedad del hecho, aspecto que quizás no fue considerado posteriormente.

Tomando en cuenta la frecuencia de las fuentes con respecto al día de publicación, los TPI se agruparon en su mayoría a partir del día miércoles, mientras que las informaciones con una sola fuente, si bien es cierto están presente todos los días, tiene un agrupamiento significativo los días "martes" con el 38,9\% de esas informaciones. La información central surgía de la emisión de los boletines oficiales de los organismos responsables, única fuente consultada por el periodista.

\section{Las fuentes informativas principales}

En general identificamos 48 fuentes citadas en los TPI. En su totalidad la frecuencia de utilización de las fuentes fue 101. No obstante, sin realizar ningún tipo de recodificación identificamos que la fuente más utilizada fue la del "Plan de Actuación Coordinada de Protección Civil" con un 17,82\%; seguida del "Instituto Geográfico Nacional" con el 12,87\%. En tercer lugar aparece "José Segura", siendo la fuente viva más utilizada (8,91\%), seguida de "Juan Carlos Carracedo" $(4,95 \%)$ y de "Nemesio Pérez" (3,96\%) 
Siguiendo la clasificación realizada por Calvo y después de la recodificación de la data obtenemos, que la utilización de las fuentes científicas estuvo únicamente limitada a "Universidades, Centros de Investigación e investigadores" con apenas siete fuentes $(14,58 \%)$ de las 48 utilizadas. Su porcentaje de utilización en los textos periodísticos fue significativo, al ser de un 45,54\%. Es de resaltar, la ausencia de la Universidad de La Laguna o de la Universidad de Las Palmas de Gran Canaria entre las fuentes citadas. No se identificaron otras fuentes relacionadas con el periodismo científico: las "Agencias informativas y de colaboraciones"; los "Organismos internacionales, congresos, embajadas, empresas"; los "Libros y revistas"; las "Bibliotecas y bases de datos".

Las principales fuentes fueron:

Plan de Actuación Coordinada de Protección Civil: El Plan de Actuación Coordinada de Protección Civil (PACPC) fue la fuente más utilizada. No es una fuente única, sino que contempla un comité de trabajo en el área presidido por el Gobierno de Canarias: "El Gobierno de Canarias, a través de la Dirección General de Seguridad y Emergencias adscrita a la Consejería de Presidencia y Justicia, la Delegación del Gobierno en las islas y el Cabildo tinerfeño han puesto en marcha un Plan de Actuación coordinada de Protección Civil ante una posible erupción volcánica en Tenerife"[54]

Es una fuente que surge desde el 9 de junio de 2004 y que comienza a consultarse a partir del mes de julio de 2004. Su mayor frecuencia fueron en las informaciones publicadas el día martes, como consecuencia de los boletines informativos que emitía los días domingo. Su mención como fuente tuvo una mayor vinculación con el tema de los "Fenómenos relacionados con la actividad volcánica". Se utilizó como única fuente en 15 textos periodísticos y en 2 textos periodísticos acompañó a otras fuentes informativas. Los textos periodísticos en los cuales estuvo presente obtuvieron una presencia significativa en la edición cubriendo de 1/12 página hasta 1 página de información.

Instituto Geográfico Nacional: Es la segunda fuente más utilizada en la cobertura informativa del hecho que nos ocupa. Es un órgano perteneciente a la Administración General del Estado, dependiente de la Subsecretaría del Ministerio de Fomento. Entre sus funciones se encuentra: "La observación, detección y comunicación de los movimientos sísmicos ocurridos en territorio nacional y áreas adyacentes" y también "la observación, vigilancia y comunicación de la actividad volcánica en el territorio nacional y determinación de los riesgos asociados"[55]

Es una fuente que se consulta desde antes de mayo de 2004. No se identificó una presencia especial en algún día de la semana. Fue una de las fuentes más utilizadas por la agencia de noticias EFE. Su mención como fuente tuvo una mayor vinculación con el tema de los "Fenómenos relacionados con la actividad volcánica". Fue una fuente que apenas fue utilizada una vez en tres registros, quiere decir que fue una fuente exclusiva en la mayoría de las ocasiones (75\%). Los textos periodísticos en los cuales estuvo presente, obtuvieron una presencia significativa en la página cubriendo de $1 / 12$ página hasta 1 página de información; aunque su mayor frecuencia fue en los TPI que ocupaban desde 1/2 de página a una página.

José Segura: Aunque es la tercera en frecuencia, es la fuente viva con mayor frecuencia de utilización en los textos periodísticos. José Segura es el Delegado del Gobierno en Canarias y coordina las actividades del Plan de Actuación Coordinada de Protección Civil.

Es una instancia que comienza a consultarse en mayo de 2004. Su mención como fuente tuvo una mayor vinculación con el tema de los "Planes de prevención, emergencia, evacuación, entre otros", el segundo tema principal de mayor importancia fue el relacionado con "Acciones y declaraciones del Gobierno de España" y es una de las fuentes utilizada en los textos periodísticos que tienen como tema principal la "Culminación del estado de alerta, semáforo en verde". Los TPI en los cuales estuvo presente obtuvieron una presencia significativa en la página cubriendo de 1/14 página hasta 1 página de información; aunque tuvo una frecuencia importante, fue en lo textos periodísticos que ocupaban desde 1/2 de página a una página donde tuvo una mayor presencia $(42,9 \%)$.

Juan Carlos Carracedo: Aunque es la cuarta fuente en frecuencia de utilización, es la segunda fuente viva con mayor frecuencia en los textos periodísticos. Juan Carlos Carracedo es volcanólogo del Consejo Superior de Investigaciones Científicas.

Su presencia es puntual en los textos periodísticos, aunque es en el mes de Junio el de mayor frecuencia. Es una fuente que cuentan con una entrevista.. No se identificó una presencia especial en algún día de la semana en particular, aunque el día "jueves" fue el de mayor frecuencia. Aunque es puntual es una fuente utilizada que fue usada por un mayor número de firmas, aunque destaca que El Día, solamente la utilizó en una oportunidad, mientras que la Agencia de Noticia EFE, la utilizó en dos oportunidades. Su mención como fuente tuvo una mayor vinculación con el tema de los "No existe crisis volcánica". Fue una fuente que apenas fue utilizada en tres oportunidades como fuente única, y en dos oportunidades compartió con otra fuente. Los textos periodísticos en los cuales estuvo presente tenían una presencia significativa en la página cubriendo de $1 / 4$ página hasta más de1 página de información.

\section{Conclusiones}

Las conclusiones que presentamos no son exhaustivas sino que abren el espacio para profundizar en el estudio del tema sobre el manejo de las fuentes en las informaciones científicas.

Con respecto a quién es el emisor fuente en la mayoría de los textos no hemos podido identificarlo, siendo por consiguiente el autor el mismo diario El Día. La investigación, y la teoría, nos señalan la conveniencia de que las informaciones periodísticas tengan un autor visible para que el lector pueda identificarlo. No hay elementos para señalar que se trate de una decisión de los periodistas de no firmar las noticias sino más bien de una política interna de El Día, por ser una característica que se repite en otros TPI. Si consideramos las noticias firmadas, aunque estos periodistas trabajan para El Día, las informaciones estuvieron mas sustentadas y se ofreció una información con más datos.

Con respecto a sí los TPI contaron o no con fuentes informativas adecuadas, debemos indicar que solamente se utilizó un solo tipo de fuente científica, es decir, aquellas que corresponden a "Universidades, centros de investigación e investigadores". La información permitía aumentar el número de tipo de fuentes, porque además existen, como la utilización de revistas especializadas, tesis de grado, bases de datos o consultas a organizaciones científicas que también se ocupa del tema volcánico. Además, hay información actualizada en bases de datos y revistas electrónicas en Internet. Las fuentes utilizadas son confiables porque reúnen las características de una fuente científica; pero fueron muy pocas para obtener otros datos para la explicación de los hechos que estaban ocurriendo. 
Esta debilidad en la variedad de las fuentes del periodismo científico se unió a la falta de contraste de fuentes, principio deontológico clave para el tratamiento de informaciones, en especial cuando su repercusión es importante. Los TPI se limitaron en su mayoría a recoger las informaciones provenientes de las instituciones oficiales y centros de investigación. Aunque el tema principal fueron, los fenómenos que estaban ocurriendo y la ausencia de una explicación sobre los mismos, fue el tema de la posibilidad de una erupción del Teide el que cobró mayor importancia. Nunca las fuentes que hablaban de los fenómenos señalaron la seguridad de una erupción; pero tampoco se explicaba qué estaba ocurriendo, dejando la posibilidad para proponer cualquier hipótesis o rumor. Fue especialmente la voz de Juan Carracedo quien manifestó el alarmismo que se estaba generando en la cobertura de la información.

Debemos señalar, que aunque en la cobertura informativa hemos identificado estas debilidades, El Día permitió que fuentes especializadas, como la de Juan Carracedo, estuvieran presentes en las páginas de opinión del diario, ofreciendo una versión menos alarmistas de los eventos que estaba ocurriendo y cuestionando la información que se estaba generando[56]. Los periodistas podían haber utilizado los artículos de opinión que se estaban publicando en el mismo diario para contrastar la información que estaban suministrando las fuentes. Inclusive en algunos editoriales de El Día se cuestionaba el tipo de información que las entidades del gobierno estaban ofreciendo[57].

Identificamos la ausencia de más entrevistas a especialistas y expertos en el área. Solamente fueron tres entrevistas las que profundizaron en el tema. Este género informativo podía haber permitido una mejor explicación de los hechos y generar tranquilidad en la población.

En esta misma línea hay una gran ausencia de la infografía como técnica informativa y de elementos multimedia. En los últimos años se ha demostrado la efectividad de estos recursos para transmitir informaciones de carácter científico, por la capacidad de síntesis y el manejo de códigos visuales que permite una explicación más eficaz del tema.

En general consideramos, que la selección de las fuentes informativas y el tratamiento que los periodistas hicieron de ella, contribuyó a crear el ambiente de alarma en la población. No porque fueran quienes originaron la alarma, porque los periodista informaron sobre los hechos que estaban ocurriendo utilizando las fuentes, sino por no contrastar la información y otorgarle a cada declaración el nivel que realmente tenía con respecto a los eventos que estaban ocurriendo. La responsabilidad del periodismo en temas científicos no se limita a ser transcriptor de declaraciones, sino que debe ofrecer una información adecuada a la realidad e importancia de los hechos.

\section{Bibliografía}

- $\quad$ Aznar, Hugo, 1999: Ética y periodismo. España: Ediciones Piados.

Belenguer Jané, Mariano, 2003: "Información y divulgación científica: dos conceptos paralelos y complementarios en el periodismo científico", en Estudios sobre el mensaje periodístico, 9.

- $\quad$ Blázquez, Niceto, 2002: La nueva ética en los medios de comunicación. Madrid: Biblioteca de Autores Cristianos.

Bonfil Olivera, Martín, 2003: Una estrategia de guerrilla para la divulgación: Difusión cultural de la ciencia. [Documento en línea] Disponible: http://www.cientec.or.cr/comunicacion/ponencias/MartinBonfil.pdf

- $\quad$ Brajnovic, Luka, 1978: Deontología periodística. España: Ediciones Universidad de Navarra.

Calvo Hernando, Manuel, 1990: Ciencia y Periodismo. Barcelona: Centro de Estudios para el Fomento de la Investigación (CEFI).

1997: Manual de Periodismo Científico. Barcelona: Bosch Casa Editorial

2002: "El Periodismo del Tercer Milenio. Problemas de la divulgación científica en Iberoamérica", en Interciencia, 27, 2, pp. 57-61

pp. $27 \overline{6-277}$

2002: "Problemas del periodismo científico en Iberoamérica", en Anuario 2002 de la AEPC.

2004: "Divulgación científica: qué es y qué no es", en Bis. Boletín Informativo del Instituto Venezolano de Investigaciones Científicas (IVIC), Junio, Disponible: http://www.ivic.ve/bis/bis132/bis.pdf

- $\quad$ Dader, José Luis, 1997: Periodismo de Precisión: Vía socioinformática de descubrir noticias. Madrid: Editorial Síntesis.

Fernández Muerza, Alex. (2002, Abril) "La comunicación científica: una necesidad social”. En: Sala de Prensa, Año IV, Vol. 2. [Documento en línea] Disponible: http://www.saladeprensa.org/art339.htm

Fundación Canaria Hospitales del Cabildo de Tenerife, 2000: Comunicación y Ciencia: II Congreso Nacional de Periodismo Científico (Comp.: Alarcó Hernández, Antonio y Meneses Fernández, María Dolores). Tenerife: Fundación Canaria de Hospitales del Cabildo.

Fundación Española Ciencia y Tecnología, 2003: Percepción Social de la Ciencia y la Tecnología en España. España: Ministerio de Ciencia y Tecnología. [Documento en línea] Disponible: http://www.campus-oei.org/salactsi/percepcion.pdf

García del Castillo, Julián, 1987: Investigación Audiovisual. Caracas: Editorial Galac.

Krippendorff, Klaus, 1990: Metodología de análisis de contenido. España: Paidós Comunicación.

López García, Guillermo, 2005: Modelos de comunicación en internet, Valencia: Tirant Lo Blanch. 
Martínez, José Luis (2001). Curso general de redacción periodística. Madrid: Paraninfo. editores.

McQuail, Denis, 1998: La acción de los medios: Los medios de comunicación y el interés público. Argentina: Amorrort 32.

Morales Matos, Guillermo, 2001: "Las Islas Canarias ¿Una región aislada”. En: Boletín de la A.G.E. (pp. 155-175), No.

Nelkin, Dorothy, 1990: "Selling sciencie" en Physics Today, Noviembre.

1990: La ciencia en el escaparate. España: Fundesco.

Nieto Olarte, Mauricio, 2002: “El público y las políticas de Ciencia y Tecnología” en Interciencia, 27.

Núñez Ladevéze, Luis, 1991: Manual para periodismo: Veinte lecciones sobre el contexto, el lenguaje y el texto de la información. Barcelona: Editorial Ariel.

- Quintanilla, Miguel Angel, 2003: "Percepción, actitud y opinión respecto de la ciencia y la tecnología SalamancaVallalodid, España". Ponencia presentada en el Primer Taller de Percepción Pública, Cultura Científica y Participación Ciudadana, realizado en Salamanca, 27 y 28 de Mayo de 2003. [Documento en línea] Disponible en: http://www.ricyt.org/interior/ normalizacion\%5Cpercepcion_publica/ 11.pdf

Reig, Ramón, 2000: Periodismo de investigación y pseudoperiodismo: Realidades, deseos y falacias. Madrid: Ediciones Libertarias.

Salaverría, Ramón, 2002: "Técnicas redaccionales para la divulgación científica", en Ramón Salaverría... [et al.], En torno al Periodismo científico: Aproximaciones. Mediatika. Cuadernos de Medios de Comunicación. Eusko Ikaskuntza / Sociedad de Estudios Vascos, San Sebastián, no 8.

- Yanes Mesa, Rafael, 2004: Géneros periodísticos y géneros anexos. Madrid: Editorial Fragua.

\section{Notas}

[1] Christie, Michael. "Welcome to Terrorife". En: Daily Record, 16 de junio de 2004

[2] Salomone, Mónica. "Crisis volcánica en Tenerife". En: El País, 22 de noviembre de 2004

[3] Pueden verse los artículos publicados por Canarias 7 (2005, Febrero 18) o el del $A B C$ (2004, Octubre 7). También el reportaje de la Nathional Geographic con el título "Canarias, cita con el fuego" (Mayo, 2004)

\section{[4] Diccionario de la Real Academia Española}

[5] "El triunfalismo que ha suscitado en el mundo científico y en la sociedad en general el Proyecto Genoma Humano podría contribuir a erigir la ciencia como el nuevo dogma de fe de la Humanidad. Pero investigadores curtidos y escépticos advierten de que ni siquiera ellos tienen todas las respuestas". Perucho, Manuel, "El día que la ciencia sea dogma". En: Diariomedico.com. [Documento en línea] Disponible: http://www.diariomedico.com/ grandeshist/numero2000/ibasica.html [Consulta: 2005, Febrero 22]

[6] Son varios los trabajos sobre el estudio de la repercusión de avances científicos en la sociedad, pero en la elaboración de nuestra investigación identificamos tres que consideramos pueden ser valiosos para presentarnos un panorama sobre su repercusión en la sociedad española. Nos referimos a los trabajos publicados en Grupo de Ciencia, Tecnología y Sociedad (CSIC), de: Plaza García, Marta y Muñoz Ruiz, Emilio (2003) "La biotecnología en la prensa española en el año 2002" [Documento en línea] Disponible: http://www.iesam.csic.es/doctrab2/dt-0316.pdf [Consulta: 2005, Abril 22]; Moreno Castro, Carolina. Luján López, José Luis y Moreno Fernández, Luis (1996) "La ingeniería genética humana en la prensa" [Documento en línea] Disponible: http://www.iesam.csic.es/ doctrab1/dt-9604.pdf [Consulta: 2005, Abril 22]; Santos, David y Muñoz, Emilio (2002) "Increasing Public Involvement in Debates on Ethical Questions of Xenotransplantation" [Documento en línea] Disponible: http://www.iesam.csic.es/doctrab2/dt-0221.pdf [Consulta: 2005, Febrero 16]

[7] "El abstracto periodismo no entra en el idioma hasta 1844 y es incluido en el diccionario académico en 1869 (Aguilar Piñal). Álvarez Barrientos encuentra la palabra periodista tardíamente, en la década de los ochenta del siglo XVIII, aunque será ya corriente durante el siglo XIX" (Ver Calvo Hernando, Manuel, 1997: Manual de Periodismo Científico. Barcelona: Bosch Casa Editorial, p. 21)

[8] Calvo Hernando, Manuel, 1997. Manual de Periodismo Científico. Barcelona: Bosch Casa Editorial, p. 16

[9] Citado por: Calvo Hernando, Manuel, 1990: Ciencia y Periodismo. Barcelona: Centro de Estudios para el Fomento de la Investigación (CEFI)

[10] Calvo Hernando, Manuel, 1990, Ob. Cit.

[11] Calvo Hernando, Manuel. (1990) Ob. Cit.

http://www.ull.es/publicaciones/latina/200709Perez_Martinez.htm 
Pérez Martínez, V. M. (2007). Algunos aspectos deontológicos en la cobertura de informaciones científicas Estu... Página 11 de 16

[12] Nieto Olarte, Mauricio, 2002: "El público y las políticas de Ciencia y Tecnología" en Interciencia, 27, 2, pp. 80-83

[13] Calvo Hernando, Manuel (1990), Ob. Cit., p. 20

[14] Belenguer Jané, Mariano, 2003: "Información y divulgación científica: dos conceptos paralelos y complementarios en el periodismo científico", en Estudios sobre el mensaje periodístico, 9, pp. 43-53

[15] Diccionario de la Real Academia Española.

[16] Belenguer Jané, Mariano, Ob.Cit. p. 46

[17] Bonfil Olivera, Martín, 2003: Una estrategia de guerrilla para la divulgación: Difusión cultural de la ciencia. [Documento en línea] Disponible: http://www.cientec.or.cr/comunicacion /ponencias/ MartinBonfil.pdf [Consulta: 2005, Febrero 24]

[18] Ver: Hernando Calvo, Manuel, 2004: "Divulgación científica: qué es y qué no es", en Bis. Boletín Informativo del Instituto Venezolano de Investigaciones Científicas (IVIC), Junio, [Documento en línea] Disponible: http://www.ivic.ve/bis/bis132/ bis.pdf [Consulta: 2005, Febrero 22]

[19] Calvo Hernando, Manuel, 2002: "El Periodismo del Tercer Milenio. Problemas de la divulgación científica en Iberoamérica", en Interciencia, 27, 2, pp. 57-61

[20] Nelkin, Dorothy, 1990: La ciencia en el escaparate. España: Fundesco, p. 27

[21] El autor plantea diferentes categorías de divulgación: Divulgación didáctica, divulgación vocacional, divulgación recreativa, divulgación democrática o social, divulgación escéptica, y divulgación periodística o periodismo científico. (Bonfil Olivera, Martín, Ob. Cit.)

[22] Martín Santos, Arantxa, 2003: "Entrevista a Javier Sampedro a propósito de la atrición de su libro: Deconstruyendo a Darwin. Los enigmas de la evolución a la luz de la nueva genética", en $A$ Parte Rei, No. 26, [Documento en línea] Disponible: http://serbal.pntic.mec.es/ cmunoz11/sampedro.pdf [Consulta: Febrero 22]

[23] Citado por Calvo Hernando, Manuel, 2002: "Problemas del periodismo científico en Iberoamérica", en Anuario 2002 de la $A E P C .$, pp. $276-277$

[24] Blázquez, Niceto, 2002: La nueva ética en los medios de comunicación. Madrid: Biblioteca de Autores Cristianos, p. XXII

[25] Brajnovic, Luka, 1978: Deontología periodística. España: Ediciones Universidad de Navarra, p. 42.

[26] García del Castillo, Julián, 1987: Investigación Audiovisual. Caracas: Editorial Galac, p. 25

[27] Krippendorff, Klaus, 1990: Metodología de análisis de contenido. España: Paidós Comunicación, p. 28.

[28] El riesgo en una región volcánica activa está siempre presente, razón por la cual Canarias cuenta con un Plan de Emergencia. (Resolución de 21 de febrero de 1996, de la Secretaría de Estado de Interior, disponiendo la publicación del Acuerdo del Consejo de Ministros por el que se aprueba la Directriz Básica de Planificación de Protección Civil ante el Riesgo Volcánico)

[29] La actividad volcánica observada en los últimos 600 años se ha producido en las islas siguientes: La Palma, Tenerife, Lanzarote y Hierro, siendo la última en 1971 con la erupción del Volcán Teneguía en La Palma. (Ver. Plan Territorial de Emergencia de la Comunidad Autónoma de Canarias)

[30] El Día, 28 de enero de 2004, p. 36.

[31] Ver, ITER, Guayota 2004, Mayo. Informativo gráfico mensual sobre la actividad sísmica y volcánica de Canarias. En: http://www.iter.es/descargas/guayota/guayota2004/GUAYOTA_Mayo_2004.jpg

[32] Canarias 7, 18 de Febrero de 2005

[33] El País, 22 de noviembre de 2004

[34] Abc, 7 de octubre de 2004

[35] Dayly Record, 16 de junio de 2004.

[36] Con el título Fue más el ruido que las nueces, la Afilarmónica Ni Fu -Ni Fa asumió la posible erupción del Teide como tema musical para las murgas del Carnaval 2005, manifestando las consecuencias de está información en la sociedad tinerfeña desde diversos puntos de vista: "Escuchen con atención, la historia de esta canción, en nada la he exagerado. Allá por Junio empezó, una posible erupción... (Ver, Santa Cruz de Tenerife, Carnaval 2005, p. 30)

[37] Ver: http://www.iter.es/l18NLayer.areasiter/es/medioambiente/infoteide/febrero05 
[39] Martínez, José Luis, 2001: Curso general de redacción periodística. Madrid: Paraninfo, p. 289.

[40] Según la Oficina de Justificación de la Difusión el diario El Día, contó entre Junio de 2003 y Junio de 2004 con un "promedio de tirada" de 28.860 ejemplares; además de un "promedio de difusión" de 22.902 ejemplares (Ver http://www.introl.es/ojdx4/diarios2.asp)

[41] La búsqueda incluyó la revisión del archivo electrónico on line del periódico (Ver http://www.eldia.es) y la revisión de los ejemplares en la hemeroteca de la Facultad de Ciencias de la Información y la hemeroteca de la Biblioteca Central, ambas de la Universidad de La Laguna. El archivo electrónico de El Día, nos permitió identificar el número de textos informativos mínimos que podíamos encontrar, permitiendo tomar una decisión sobre la necesidad o no de seleccionar una muestra. En la revisión física de los ejemplares pudimos corroborar que hay una relación entre el número de textos informativos de la versión digital y la impresa. No obstante, el sistema electrónico planteó algunas debilidades que exigió la revisión de los ejemplares físicos: 1) La versión digital no incluye las primeras páginas, los sumarios ni las últimas páginas; 2) El tipo de diagramación del archivo electrónico no mantiene las mismas características que la edición impresa; 3) Hay recursos gráficos (mapas, fotos, infografías) que no se encuentra en el archivo electrónico. No obstante, el estudio estaba centrado en la publicación digital teniendo como soporte la edición impresa para identificar posibles diferencias.

\section{[42] Yanes Mesa, Rafael, 2004: Géneros periodísticos y géneros anexos. Madrid: Editorial Fragua p. 43.}

[43] "El Terremoto del Océano Índico de 2004 fue un sismo submarino con una magnitud de 9.2 que hizo temblar el este del Océano Índico el 26 de diciembre de 2004 (a las 00:58 UTC, o 07:58 en el tiempo local de la región). El terremoto, que se originó en el mar cerca de la costa norteña de la isla indonesia de Sumatra, provocó varios tsunamis masivos que afectaron a áreas costeras de ocho países asiáticos y causaron la muerte de aproximadamente 288.000 personas. Este fue el quinto terremoto más fuerte de la historia moderna (desde que se registran con sismógrafos)" (Wikipedia: http://es.wikipedia.org/wiki/ Terremoto_del_Oc\%C3\% A9ano_\%C3\%8Dndico_de_2004

[44] Desde el principio de nuestra investigación sabíamos que nuestras unidades de análisis eran los textos periodísticos informativos (TPI); pero con la finalidad de evaluar la importancia que El Día le otorgó al tema de estudio registramos inicialmente aquellos textos periodísticos editoriales (TPE), es decir los que fueron publicados en la Primera Página, Sumario y Última Página del diario y que también fueron recogidos en la versión digital. Los resultados señalan que efectivamente $E l$ Día valoró las informaciones que provenían de los eventos que estaban ocurriendo alrededor del Teide. En un total de 63 registros sobre el tema, el $15,9 \%$ correspondió a TPE, el resto $84,1 \%$ a TPI, que fueron los textos estudiados. Otro indicador de la importancia del tema fue la ubicación de los TPE en El Día: En seis ocasiones la información ocupó la portada del periódico y en cuatro ocasiones el "Sumario".

[45] El Día, 28 de enero de 2004

[46] El Día, 3 de abril de 2004

[47] El Día, 2 de mayo de 2004

[48] El Día, 10 de mayo de 2004

[49] El Día, 11 de mayo de 2004

[50] El Día, 20 de mayo de 2004

[51] El Día, 20 de mayo de 2004

[52] Yanes Mesa, Rafael. Ob. Cit., p. 57

[53] Yanes Mesa, Rafael. Ob. Cit., p. 60

[55] Información en la Sitio Web oficial del Instituto Geográfico Nacional: http://www.mfom.es/ign/

[56] Ver por ejemplo los artículos de Juan Carracedo: "El volcán de octubre de 2004" publicado en El Día, Domingo 13 de Junio de 2004 o el artículo "La predicción de erupciones volcánicas y los juegos de azar" publicado en El Día, Domingo 31 de octubre de 2004.

[57] Ver editoriales de El Día: Domingo, 24 de octubre de 2004 y Domingo, 14 de noviembre de 2004.

\section{FORMA DE CITAR ESTE TRABAJO EN BIBLIOGRAFÍAS, SEGÚN LA APA}

Pérez Martínez, V. M. (2007). Algunos aspectos deontológicos en la cobertura de informaciones científicas Estudio de caso: "Crisis volcánica" del Teide . Revista Latina de Comunicación Social, 62. Recuperado el x de xxxx de 200x, de http://www.ull.es/publicaciones/latina/200709Perez_Martinez.htm 